TABLE 1. Survey Responses

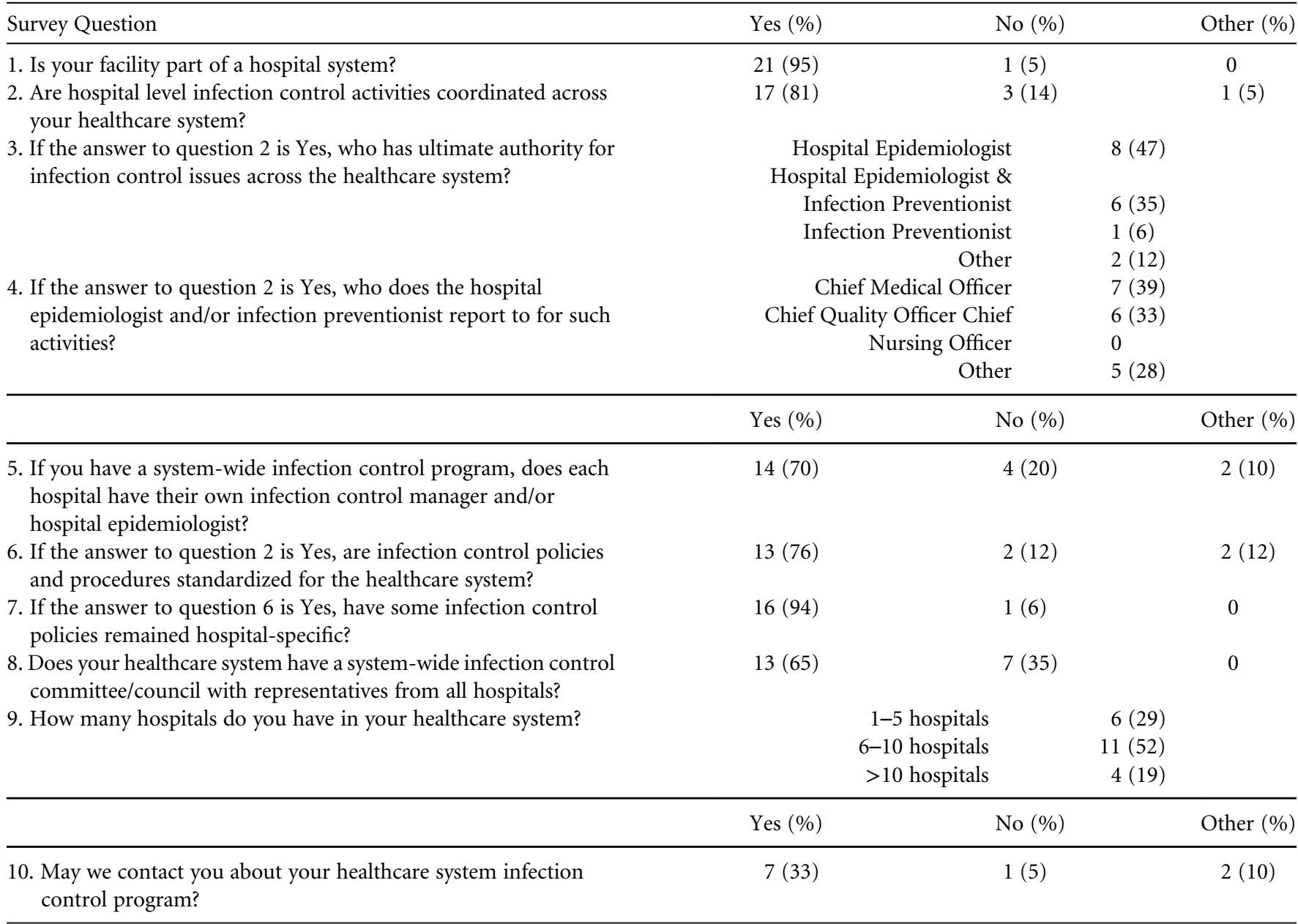

\section{Adherence to Central-Line Insertion Practices (CLIP) with Peripherally Inserted Central Catheters (PICC) and Central Venous Catheters (CVC): A Prospective Study of 50 Hospitals in China}

To the Editor - Two types of common central venous central lines (CL), peripherally inserted central catheters (PICC) and central venous catheters (CVC), are widely applied in the care of patients with severe diseases treated in surgical, intensive care, oncological and hematological units. ${ }^{1,2}$

In addition, invasive procedure for PICC and CVC could result in central-line-associated bloodstream infections (CLABSIs), leading to significant morbidity, mortality, and cost among hospitalized patients. ${ }^{3}$ Strict compliance with central-line insertion practices (CLIP), including hand hygiene, proper use of a skin antiseptic prior to insertion, skin preparation agent completely dried before insertion, and application of maximal sterile barriers (MSB) during insertion, ${ }^{4}$ would significantly decrease the risk of subsequent CLABSIs. ${ }^{5}$ However, CLIP adherence in China has not been reported. In this study, we first performed a prospective multicenter study using a cell-phone app (cf, software application) to assess CLIP adherence for PICC and CVC insertions in Jiangsu Province in China from March 1 through March 31, 2017. During the study period, 12 of 13 cities (92.3\%) and 50 general hospitals were included in this study. WeChat (www.Tencent.com) and contact groups were applied to train all the investigators online to achieve homogeneity. All investigators observed all PICC and CVC procedures and uploaded the data to the terminal database via an app installed in a cell phone.

This study included 1,377 PICC insertions (tertiary general hospitals, 1,148 insertions, $83.4 \%$; secondary general hospitals, 229 insertions, 16.6\%) and 2,304 CVC insertions (tertiary general hospitals, 1,980 insertions, $85.9 \%$; secondary general hospitals, 324 insertions, 14.1\%). Compared to CVC insertions, adherence to hand hygiene and complete drying of the skin disinfectant before insertion were significantly greater with PICC insertions $(P<.001)$ (Table 1$)$. In addition, CVCs were always placed by doctors, while PICCS were mainly 
table 1. Adherence to Central Line Insertion Practices (CLIP) Between PICC and CVC

\begin{tabular}{|c|c|c|c|c|c|c|}
\hline \multirow[b]{2}{*}{ Variable } & \multicolumn{2}{|c|}{ PICC } & \multicolumn{2}{|c|}{$\mathrm{CVC}$} & \multirow[b]{2}{*}{ Statistics } & \multirow[b]{2}{*}{$P$ Value } \\
\hline & $\begin{array}{c}\text { No. } \\
(\mathrm{N}=1,377)\end{array}$ & $\begin{array}{c}\text { Adherence, } \\
\%\end{array}$ & $\begin{array}{c}\text { No. } \\
(\mathrm{N}=2,304)\end{array}$ & $\begin{array}{c}\text { Adherence, } \\
\%\end{array}$ & & \\
\hline Hand hygiene performed before insertion & 1,374 & 99.8 & 1,880 & 81.6 & 277.945 & $<.001$ \\
\hline Appropriate skin prep before insertion & 1,377 & 100.0 & 2,304 & 100.0 & NA & NA \\
\hline Skin prep agent completely dried before insertion & 1,368 & 99.4 & 1,836 & 79.7 & 295.318 & $<.001$ \\
\hline $\begin{array}{l}\text { Maximal sterile barriers (MSB) used before } \\
\text { insertion }\end{array}$ & 1,267 & 92.0 & 1,161 & 50.4 & 664.978 & $<.001$ \\
\hline Sterile gloves & 1,364 & 99.1 & 2,278 & 98.9 & 0.28 & .597 \\
\hline Sterile gown & 1,297 & 94.2 & 1,267 & 55.0 & 626.556 & $<.001$ \\
\hline Cap & 1,349 & 98.0 & 2,244 & 97.4 & 1.203 & .273 \\
\hline Mask & 1,357 & 98.7 & 2,259 & 98.1 & 1.246 & .264 \\
\hline Large sterile drape & 1,360 & 98.8 & 1,768 & 76.7 & 327.637 & $<.001$ \\
\hline
\end{tabular}

NOTE. CLIP, central-line insertion practices; PICC, peripherally inserted central catheter; CVC, central venous catheter; NA, not available; Statistics, Pearson Chi-square test.

placed by nurses. Our results suggest better adherence with CLIP by nurses than by doctors.

It has been reported that the application of maximal sterile barriers (MSBs) used before CVC insertion could lower medical costs and decrease the incidence of catheter colonization, catheter-related bloodstream infections, and death, ${ }^{6}$ which reflects the importance of MSBs in the prevention of CLABSI. However, compliance with MSB protocols for CVC insertions was only $50.4 \%$ in this investigation, especially for sterile gowns (55.0\%) and large sterile drapes (76.7\%). This finding suggests that enough and timely access to adequate supplies and personal protective equipment (PPE) for CLABSI prevention would greatly affect compliance with CLIP, and these costs should be fully supported in departmental budgets. In stratified analyses by department, the worst compliance with MSB use in CVC insertion occurred for anesthesia and operating rooms (1.6\%), radiotherapy units $(40 \%)$, and neurosurgery units $(47.6 \%)$, which strongly suggests the need to improve supervision, to strengthen training, and to increase feedback in the use of MSBs.

In conclusion, our data indicate that compared to PICC insertions, there was significantly less adherence with hand hygiene, complete drying of the skin disinfectant, and poor adherence with MSBs with CVC insertions. CLIP adherence should also be monitored daily to optimize patient safety.

\section{ACKNOWLEDGMENTS}

Financial support: This study was supported by grants from the 2017 Hospital Management Innovation Research Project (grant no. JSYGY-2-2017-205) and the Priority Academic Program Development of Jiangsu Higher Education Institutions (PAPD grant no. JX10231801).

Potential conflicts of interest: All authors declare that they have no competing interests.

\author{
Wensen Chen, MBBS, MPH; ${ }^{1}$ \\ Yiqun Yang, $\mathrm{MPH}{ }^{2}$ \\ Huifen Li, BSN; ${ }^{1}$
}

\section{Xiaoqiang Huang, $\mathrm{MD}{ }^{3}$ Weihong Zhang, $\mathrm{MD}^{1}$}

Affiliations: 1. Infection Management Office, the First Affiliated Hospital of Nanjing Medical University, Nanjing, Jiangsu, China; 2. Department of Nursing, the First Affiliated Hospital of Soochow University, Suzhou, Jiangsu, China; 3. Department of Infection Management, the Affiliated Xiaolan Hospital, Southern Medical University, Xiaolan People's Hospital, Zhongshan City, Guangdong, China.

Address correspondence to Zhang Wei-Hong, First Affiliated Hospital, Nanjing Medical University, 300 Guangzhou Rd, Nanjing, Jiangsu, China (wensenchen@njmu.edu.cn).

Infect Control Hosp Epidemiol 2018;39:122-123

(c) 2017 by The Society for Healthcare Epidemiology of America. All rights reserved. 0899-823X/2018/3901-0024. DOI: 10.1017/ice.2017.259

\section{REFERENCES}

1. Johansson E, Hammarskjöld F, Lundberg D, Arnlind MH. Advantages and disadvantages of peripherally inserted central venous catheters (PICC) compared to other central venous lines: a systematic review of the literature. Acta Oncol 2013;52:886-892.

2. Guembe M, Pérez-Granda MJ, Capdevila JA, et al. Nationwide study on peripheral-venous-catheter-associated-bloodstream infections in internal medicine departments. J Hosp Infect 2017;97:260-266; pii:S0195-6701(17)30390-0.

3. Furuya EY, Dick AW, Herzig CT, Pogorzelska-Maziarz M, Larson EL, Stone PW. Central line-associated bloodstream infection reduction and bundle compliance in intensive care units: a national study. Infect Control Hosp Epidemiol 2016;37:805-810.

4. Surveillancefor Central Line Insertion Practices (CLIP) Adherence. Centers for Disease Control and Prevention (CDC) website. https:// www.cdc.gov/nhsn/acute-care-hospital/clip/index.html. Updated 2017. Accessed March 10, 2017.

5. O'Grady NP, Alexander M, Burns LA, et al. Guidelines for the prevention of intravascular catheter-related infections. Clin Infect Dis 2011;52:e162-e193.

6. Hu KK, Veenstra DL, Lipsky BA, Saint S. Use of maximal sterile barriers during central venous catheter insertion: clinical and economic outcomes. Clin Infect Dis 2004;39:1441-1445. 